\title{
Starch/Poly (Glycerol-Adipate) Nanocomposite Film as Novel Biocompatible Materials
}

\author{
Domenico Sagnelli ${ }^{1, *}$, Robert Cavanagh ${ }^{2}$, Jinchuan Xu ${ }^{1,3,4}{ }^{1}$, Sadie M. E. Swainson ${ }^{2}$, \\ Andreas Blennow ${ }^{3}{ }^{\circledR}$, John Duncan ${ }^{5}$, Vincenzo Taresco ${ }^{1}$ and Steve Howdle ${ }^{1}$ \\ 1 School of Chemistry, University of Nottingham, University Park, Nottingham NG7 2RD, UK \\ School of Pharmacy, University of Nottingham, University Park, Nottingham NG7 2RD, UK \\ Department of Plant and Environment Sciences, University of Copenhagen, 1871 Frederiksberg C, Denmark \\ School of Food Science and Engineering, South China University of Technology, Guangzhou 510640, China \\ 5 Lacerta Technology, Shepshed, Loughborough LE12 9GX, UK \\ * Correspondence: domenico.sagnelli@nottingham.ac.uk
}

Received: 11 July 2019; Accepted: 27 July 2019; Published: 30 July 2019

check for

\begin{abstract}
Starch is one of the most abundant polysaccharides on the earth and it is the most important source of energy intake for humans. Thermoplastic starch (TPS) is also widely used for new bio-based materials. The blending of starch with other molecules may lead to new interesting biodegradable scaffolds to be exploited in food, medical, and pharmaceutical fields. In this work, we used native starch films as biopolymeric matrix carriers of chemo enzymatically-synthesized poly (glycerol-adipate) (PGA) nanoparticles (NPs) to produce a novel and biocompatible material. The prototype films had a crystallinity ranging from $4 \%$ to $7 \%$. The intrinsic and thermo-mechanical properties of the composite showed that the incorporation of NPs in the starch films decreases the glass transition temperature. The utilization of these film prototypes as the basis for new biocompatible material showed promise, particularly because they have a very low or even zero cytotoxicity. Coumarin was used to monitor the distribution of the PGA NPs in the films and demonstrated a possible interaction between the two polymers. These novel hybrid nanocomposite films show great promise and could be used in the future as biodegradable and biocompatible platforms for the controlled release of amphiphilic and hydrophobic active ingredients.
\end{abstract}

Keywords: starch; nanoparticles; biomaterial; biocompatible; Caco-2; polyglycerol adipate; DMA; composites; polymer synthesis

\section{Introduction}

Starch is one of the most abundant polysaccharides on the earth and has a huge potential as a very well-defined raw material for functionalized biomaterials. Starch consists of two major polymers, amylose and amylopectin [1]. Amylopectin is a much larger molecule than amylose $\left(M_{\mathrm{w}}=1 \times 10^{7}-1 \times 10^{9}\right)$ and represents $65 \%-75 \%$ of starch. It has a highly branched structure, with $\alpha-(1-4)$-linked D-glucose backbones and approximately 5\% of $\alpha-(1-6)$-linked branches [2]. Amylose is a quasi-linear polymer composed by $\alpha-(1-4)$-linked glucose residues and has a molecular weight of approximately $1 \times 10^{5}$ to $1 \times 10^{6} \mathrm{Da}$ [3]. Amylose has a small degree of $\alpha$-(1-6)-linked branches. Amylopectin and amylose together form semi-crystalline and insoluble granules with an internal lamellar structure.

Thermoplastic starch (TPS) has been widely used as raw material for the production of novel biobased compostable materials [4-8]. Unfortunately, TPS forms a brittle material that is sensitive to water [8]. An option to improve the intrinsic characteristics of TPS is to blend or coat it with other 
biocompatible and bio-renewable polymers. Depending on the polymer used, the blend will have diverse functionalities and properties $[4,9,10]$.

Bio-based poly (glycerol-adipate) (PGA) can be envisaged as a suitable candidate for starch co-polymeric-blends. In fact, PGA is a functionalizable, biocompatible, and biodegradable macromolecule [11-13]. Interestingly, PGA can be synthesized by a green poly-condensation reaction catalyzed by a lipase immobilized on acrylic beads (Novozyme 435) (Figure 1).

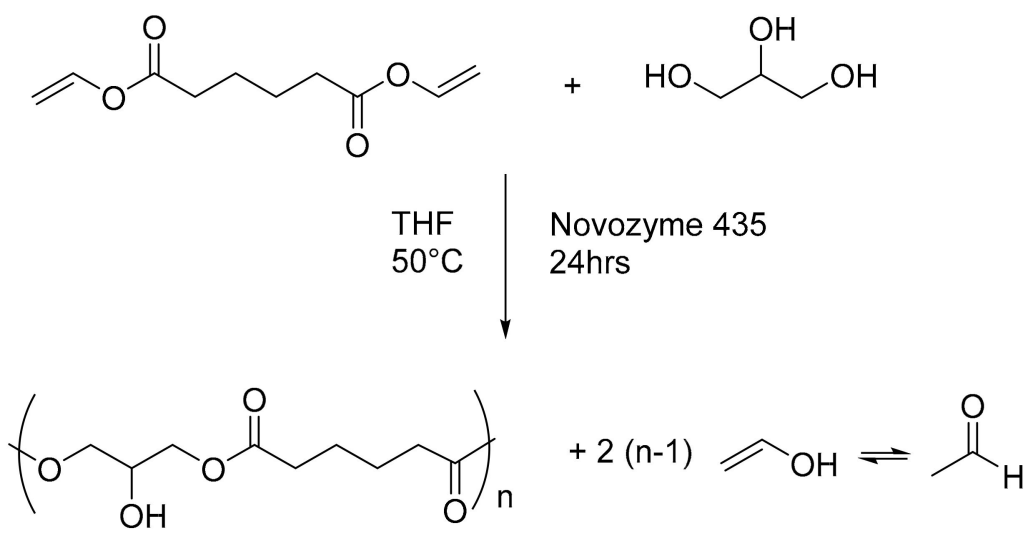

Figure 1. Polycondensation reaction of glycerol and dyvinil adipate catalyzed by Novozyme 435.

The enzymatic synthesis of poly-(glycerol adipate) (PGA) from divinyl adipate and glycerol is a simple and versatile strategy [11-13]. It is possible to exploit the chemo- and regio-selectivity of the enzyme to leave the secondary hydroxyl moiety unreacted, avoiding tedious and complicated protection/deprotection chemistry steps [12].

The reaction temperature is optimally performed at $50{ }^{\circ} \mathrm{C}$ to produce a highly linear polymer backbone motif with negligible branching [14-17]. Since the hydroxyl moieties are positioned in the outer shell of the nanoparticles (NPs), PGA will create low-force interactions with starch. This will avoid phase separations and allow the formation of a cohesive film. Furthermore, the utilization of a polysaccharide as the matrix for PGA nanoparticles will enable the formation of a possible biocompatible device for drug or flavor delivery.

In the present study, native starch extracted from barley was used as a base for cast biomaterial films. Because of the intrinsic brittleness of starch, the films were plasticized with glycerol. Furthermore, native starch films were used as a coating of PGA nanoparticles to produce a novel and biocompatible scaffold. The materials produced were characterized for their macro- and microstructure, chemical composition, crystallinity, thermos-mechanical properties, and biocompatibility.

\section{Materials and Methods}

\subsection{Materials}

Unless otherwise stated, all the chemicals used in this work were purchased from Sigma-Aldrich. Barley starch was kindly donated by Altia starch (Sweden). The human intestinal epithelial adenocarcinoma cell line, Caco-2, were obtained from the American Type Culture Collection (ATCC) and used between passages 45 and 50. Cells were cultured in Dulbecco's modified Eagle medium (DMEM) supplemented with $10 \%$ fetal bovine serum (FBS) at $37^{\circ} \mathrm{C}$ in a humidified incubator with $5 \%$ $\mathrm{CO}_{2}$. Cells were routinely grown in $75 \mathrm{~cm}^{2}$ culture flasks to $70 \%$ confluence. Caco-2 cells were seeded at a density of $1 \times 10^{4}$ cells per well in 96-well plates for cytotoxicity experiments and cultured for $24 \mathrm{~h}$ before assaying. 


\subsection{Methods}

\subsubsection{Poly-(Glycerol Adipate)(PGA) Synthesis}

Glycerol (125 mmol) and divinyl adipate $(125 \mathrm{mmol})$ were poured into a $250 \mathrm{~mL} 3$-neck round-bottomed flask and mixed with anhydrous $50 \mathrm{~mL}$ tetrahydrofuran (THF). The resulting system was heated in a tared oil bath. Lipase $(1.1 \mathrm{~g}$ extracted $\geq 5000 \mathrm{U} / \mathrm{g}$, recombinant, expressed in Aspergillus niger) was added into the mixture when the internal temperature of the reaction reached 40 to $45^{\circ} \mathrm{C}$ and achieved a single homogeneous liquid phase. The stirring was set at $250 \mathrm{rpm}$ using an overhead mechanical stirrer for $24 \mathrm{~h}$. The reaction was stopped by filtration of the enzyme beads and THF evaporation. Any residual free enzyme in the dry fraction was deactivated by heating the product at $95^{\circ} \mathrm{C}$ for $1 \mathrm{~h}$. The final product was a pale yellow highly viscous liquid [12].

\subsubsection{PGA N-Acyl-Tyrosine (Pgatyr) Coupling Reaction}

A simple and well-established Steglich esterification was adopted to couple N-Acyl-Tyrosine to the free hydroxyl groups of PGA, aiming at 30\% substitution of the total of the $\mathrm{OH}$ functionalities (Figure 2). Briefly, PGA ( $4.95 \mathrm{mmol})$ and DMAP $(0.15 \mathrm{mmol})$ were added to anhydrous THF $(20 \mathrm{~mL})$ at room temperature in a round bottom flask under magnetic stirring until complete dissolution of the solids. Another THF solution (final volume $20 \mathrm{~mL}$ ) was prepared by dissolving $1.20 \mathrm{mmol}$ of DCC and $1.49 \mathrm{mmol}$ of $\mathrm{N}$-acyl Tyr. The two solutions were mixed and left to stir overnight under $\mathrm{N}_{2}$ atmosphere. The resulting dicyclohexylurea insoluble precipitate was removed by centrifugation. The modified polymer was precipitated in a solution $0.1 \mathrm{M} \mathrm{NaOH}$ and twice in cold $\mathrm{MeOH}$. The residual material was dried under reduced pressure to a stable weight.

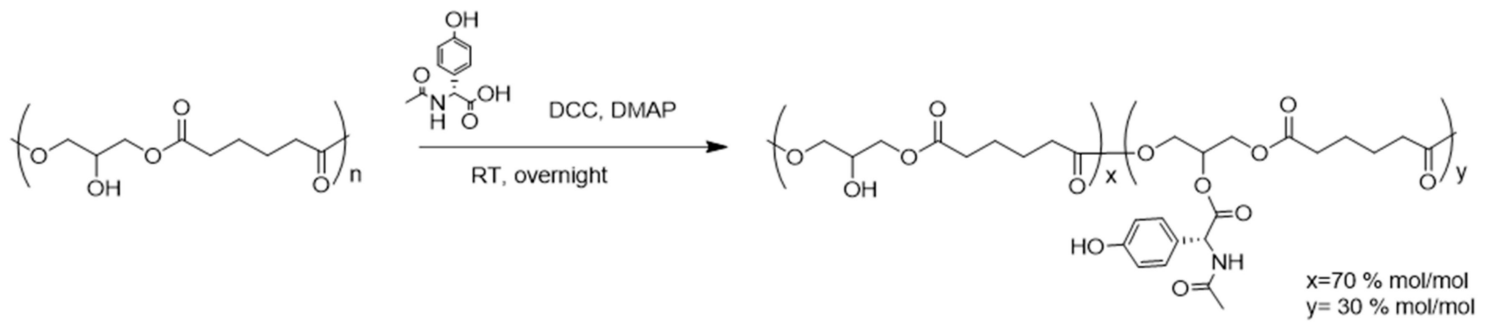

Figure 2. Coupling reaction scheme of Poly-(Glycerol Adipate)(PGA) and tyrosine.

\subsubsection{Particle Size of PGA and PGATyr Nanoparticles (NPs)}

PGA and PGATyr NPs were produced by the nanoprecipitation technique, reaching a final concentration of $1.5 \mathrm{mg} / \mathrm{mL}$ in the aqueous medium. Initially, the polymers were dissolved in acetone $(2 \mathrm{~mL})$ and added to ultrapure water $(10 \mathrm{~mL})$ under constant magnetic stirring (550 rpm) at Room Temperature (RT) $\left(19^{\circ} \mathrm{C}\right)$. The organic solvent was evaporated from the mixture while being agitated in a fume hood. Particle sizes were measured by dynamic light scattering (DLS, Zetasizer Nano ZS, Malvern Instruments, Malvern).

\subsubsection{Enzymatic Degradation Assay}

To evaluate the biodegradability of PGA and PGATyr, NPs (initial concentration $1.5 \mathrm{mg} / \mathrm{mL}$ ) were diluted in PBS at a final concentration of $0.5 \mathrm{mg} / \mathrm{mL}$. Lipase was prepared in PBS (pH 7.4) to a concentration of $10 \mathrm{mg} / \mathrm{mL}$ ( $215 \mathrm{units} / \mathrm{mL}$ ). In total, $50 \mu \mathrm{L}$ of the lipase mixture was added to the NP suspensions. DLS measurements were carried out for $180 \mathrm{sec}$ after the enzyme addition at constant shaking at $37^{\circ} \mathrm{C}$ to evaluate the swelling of the NPs in degradative conditions.

\subsubsection{Melt Behavior Measurements}

The melting behavior of the starch used in this study was measured using a high-pressure rheometer (Anton-Paar MCR series, Graz, Austria). In particular, a stirrer geometry was used and the rheometer 
program was as follows: $960 \mathrm{rpm}$ for $90 \mathrm{~s}$ at $30^{\circ} \mathrm{C}$, followed by a pasting step at $170 \mathrm{rpm}$, a temperature ramp from 37 to $95^{\circ} \mathrm{C}$ with a heating rate of $1^{\circ} \mathrm{C} / \mathrm{min}$, an isotherm at $95^{\circ} \mathrm{C}$ for $5 \mathrm{~min}$, a final ramp from 95 to $50{ }^{\circ} \mathrm{C}$ at a cooling rate of $5{ }^{\circ} \mathrm{C} / \mathrm{min}$, for $5 \mathrm{~min}$, and a final isotherm at $50^{\circ} \mathrm{C}$ for $5 \mathrm{~min}$.

\subsubsection{Water Contact Angle $(\Theta w)$}

Water contact angles $(\Theta w)$ were measured at room temperature by employing a KSV Cam 200 (KSV Instruments Ltd, Helsinki, Finland) and angle pictures were analyzed with dedicated software (CAM200 1.1-KSV instruments). Four measurements were recorded for each polymer.

\subsubsection{ATR FT-IR}

ATR-IR spectra were acquired by means of an attenuated total reflection spectrophotometer (Agilent Technologies Cary 630 FTIR, Santa Clara CA, USA) equipped with a diamond single reflection ATR unit. Spectra were acquired with a resolution of $4 \mathrm{~cm}^{-1}$, in the range $4000-650 \mathrm{~cm}^{-1}$ by acquiring 32 interferograms. Spectra were analyzed with the open access software SpectraGryph1.2.

\subsubsection{Casting of Films}

The native water content (\%w) of starch (ST) was measured by thermogravimetric analysis and was taken into account for the final formulation. The films were casted with a final surface area of $10 \mathrm{mg} / \mathrm{cm}^{2}$. The PGA nanoparticles were, respectively, $3 \%$ and $10 \%$ of starch (dry weight, d.w.). To achieve plasticization of the films, $25 \%$ of glycerol was included to the mixture.

Starch was suspended in de-ionized water. The solutions were mixed at $300 \mathrm{rpm}$ with magnetic stirring following heating in an oil bath at $95^{\circ} \mathrm{C}$ until gelatinization. After the gelatinization phase, the solutions were placed into an ice bath while being agitated. When a temperature of $50{ }^{\circ} \mathrm{C}$ was reached, glycerol and PGA NPs were added and mixed until full homogenization. The solutions were immediately casted in Teflon-coated petri dishes and the films dried at $50^{\circ} \mathrm{C}$ in an oven with ventilation. Prior to any analysis, the film prototypes were equilibrated to stabilize the moisture content in a desiccator containing a saturated solution of potassium chloride (relative humidity $85 \% \pm 1 \%$ ).

\subsubsection{X-Ray Scattering}

The films were loaded in a sealed holder to minimize evaporation during the measurement. X-ray measurements were conducted using a Panalytical Xpert Pro (Nottingham, UK).

The radially averaged intensity is given as a function of the scattering vector, $q=4 \pi \sin \theta / \lambda$, where $\lambda$ is the wavelength and $2 \theta$ is the scattering angle. The samples were measured in the WAXS setting (wide-angle $X$-ray scattering) to an upper $2 \theta$ value of $35^{\circ}$ or $2.86 \AA$. The exposure time was $400 \mathrm{~s} / \mathrm{step}$ with a step size of $\left(0.0131303^{\circ}\right)$ [5].

\subsubsection{Dynamic Mechanical Analysis}

Dynamic mechanical analysis (DMA) with a temperature gradient was performed in tension mode with a displacement of $0.005 \mathrm{~mm}$ and frequencies of 1 and $10 \mathrm{~Hz}$. A standard heating rate of $3{ }^{\circ} \mathrm{C}$ $\mathrm{min}^{-1}$ was used and a ramp from -50 to $120^{\circ} \mathrm{C}$. The experiments were performed on prototypes with a length of $10 \mathrm{~mm}$ [7]. The glass transition temperature was estimated by comparing the derivative function of the storage modulus and the $\tan$ delta $(\tan \delta)$ peak.

For measurements in a humidity-controlled environment, the temperature was kept constant at $37^{\circ} \mathrm{C}$ and the relative humidity (RH) was increased up to $80 \%$ at a rate of $0.3 \% \mathrm{RH} / \mathrm{min}$ and kept at $80 \%$ until equilibration.

\subsubsection{Confocal Microscopy}

Confocal laser scanning microscopy (CLSM) was performed using a Leica system (Leica SP5-X, Leica Microsystems) equipped with x63 water immersion objectives. Films were prepared as previously 
described. PGA and PGA-Tyr NPs were prepared by nanoprecipitation to a final concentration of $1.5 \mathrm{mg} / \mathrm{mL}$. Coumarin 30, used as fluorophore, was dissolved in methanol to a concentration of $1 \mathrm{mg} / \mathrm{mL}$ and the polymer was dissolved in acetone at the same concentration. Coumarin 30 solution $(15 \mu \mathrm{L}$ per mg of polymer) was added to the polymer solution and the resultant solution added dropwise to ultrapure water under magnetic stirring. The nanoparticle suspension was immediately transferred to a dialysis membrane with a $3.5 \mathrm{kDa}$ MWCO (Mw cut off, Regenerated Cellulose Dialysis Tubing, Type T1, Fisherbrand) and dialyzed against water for $4 \mathrm{~h}$ to remove the acetone, methanol, and any free coumarin 30. The acetone was evaporated, and particles were dialyzed against water for $3 \mathrm{~h}$. Images were analyzed with the LAS X 3.0 software.

\subsubsection{Cytotoxicity Test}

Assessment of cytotoxicity was performed in accordance with ISO standard 10993-5 via an extract test to evaluate the biocompatibility of any leachable material by-products by the simulation of clinical application [18]. The PrestoBlue cell viability assay (ThermoFisher) was used to probe mitochondrial function and the detection of lactose dehydrogenase (LDH) release was used to measure cell membrane integrity (Sigma-Aldrich, TOX7 kit, St. Louis MO, USA). Films were UV sterilized for $20 \mathrm{~min}$ and placed in DMEM (Dulbecco minimal essential medium) with $10 \%$ fetal bovine serum (FBS) $\left(3 \mathrm{~cm}^{2}\right.$ per ml) for 24,48 and $72 \mathrm{~h}$ and incubated at $37^{\circ} \mathrm{C}$ in a humidified incubator with $5 \% \mathrm{CO}_{2}$. The resulting solutions were applied to cells (100 $\mu \mathrm{L}$ per well) and incubated for $48 \mathrm{~h}$. A negative control with DMEM and a positive control $(0.1 \%$ Triton $\mathrm{X}-100)$ were also included in the cytotoxicity experiments. Following exposure, $50 \mu \mathrm{L}$ of supernatant were collected per well for analysis of LDH content. Cells were washed twice with phosphate-buffered saline (PBS) and $100 \mu \mathrm{L} 10 \%$ (v/v) PrestoBlue reagent diluted in DMEM

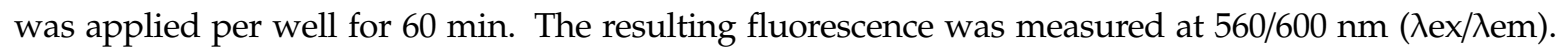
Relative metabolic activity was calculated by setting values from the negative control as $100 \%$ and positive control values as $0 \%$ metabolic activity. Assessment of LDH release was performed according to the manufacturer's instructions and involved adding $100 \mu \mathrm{L}$ of LDH reagent to the collected supernatant samples and incubating them at room temperature shielded from light for 25 minutes. Absorbance was measured at $492 \mathrm{~nm}$. Relative LDH release was calculated with the negative control absorbance at $492 \mathrm{~nm}$ taken as $0 \%$, and the positive control, assumed to cause total cell lysis, as $100 \%$.

\section{Results and Discussion}

\subsection{Native Starch Melt and Crystallinity Characterization}

Before the production of the films, the native starch was analyzed for its melting behavior in water to allow a precise design of the melting process. In particular, the $10 \%$ suspension of starch granules was processed using a high-pressure sealed rheometer to avoid any evaporation of water (Figure 3). This rheometer allowed a better understanding of the starch behavior during melting as compared to standard rapid visco analysers, which have open vessels, thus risking significant water evaporation.

The measurement showed a pasting viscosity of $18.8 \mathrm{cP}$ at a temperature of $88^{\circ} \mathrm{C}$. The hot peak viscosity was measured at $95{ }^{\circ} \mathrm{C}$. During the isotherm, a breakdown of viscosity was detected at $95^{\circ} \mathrm{C}$, indicating complete melting of the starch. During the fast cooling phase, when a temperature of 50 ${ }^{\circ} \mathrm{C}$ was reached, the melt started to form a gel, with a peak viscosity of $1300 \mathrm{cP}$. These measurements allowed the design of a melting profile for the production of the films [6].

Since starch has a semi-crystalline nature, it was first characterized using wide angle X-ray scattering (WAXS) to identify its diffraction pattern following full hydration [5]. The initial crystallinity also provided data to calculate the degree of disruption of the native crystallinity in the films. The results showed that the native starch possessed an average degree of crystallinity $(20 \%)$ and was found to be a common mixture of A-type and B-type polymorphs, with a V-type peak centered at 20 (20) [19]. These data prove that the starch used, even if it was industrially extracted, did not undergo any transformation of the crystalline and amorphous structure. 


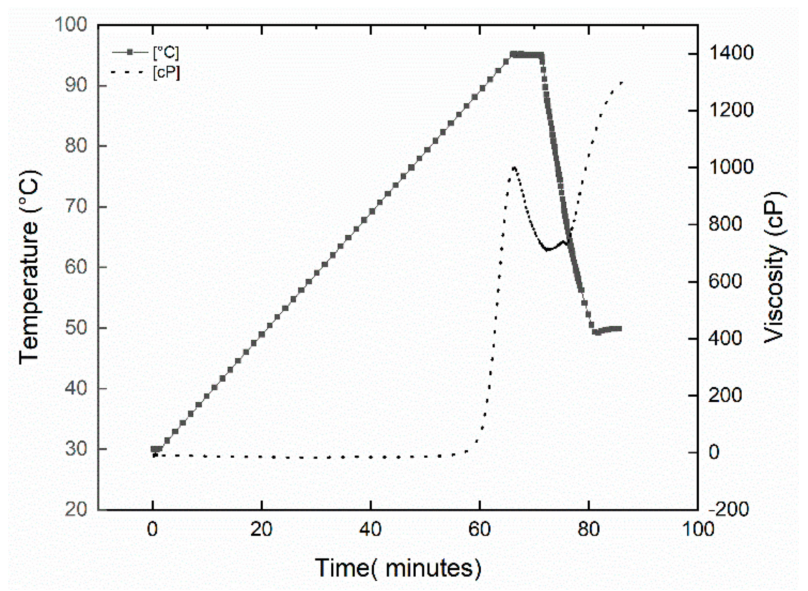

Figure 3. Melting profile measurement of $10 \%$ starch suspension using a high-pressure rheometer.

\subsection{Synthesis and Characterization of Nanoparticles}

PGA and PGATyr NPs were formulated via the simple nanoprecipitation technique, using acetone as the organic phase. Due to the intrinsic amphiphilic structure established among the backbone and the side groups of these polymers, the poly-(glycerol adipate) can favorably self-assemble in water without the use of surfactants, thus reducing undesired side effects. Before the preparation of the composite films, an enzymatic dynamic light scattering (DLS) screening assay to confirm the biodegradability of PGA and PGATyr NPs was performed [20]. PGATyr NPs (145 nm) were smaller than the NPs produced from bare PGA $(175 \mathrm{~nm})$, confirming the existence of better packing in the hydrophobic core, supposedly due to the $\pi-\pi$ interactions among the aromatic moieties [13].

NPs' size alterations were recorded in the presence of lipase against NPs enzyme-free controls. It has been suggested that the increase in particle size represented by the swelling of the NPs is a sign of degradation when interacting with enzymes [21]. Remarkable swelling in terms of hydrodynamic size was observed for both materials. However, a different swelling pattern was observed for the two polyesters after $3 \mathrm{~h}$ of contact with lipase (Figure 4). In fact, PGATyr reached half of the size of the pure PGA NPs. These differences may be related to a lower enzyme accessibility because of steric hindrance caused by different polymer chains spatial arrangement or chains packing and interactions linkable to the $\pi-\pi$ stacking interactions amongst the aromatic tyrosyl residues [13].

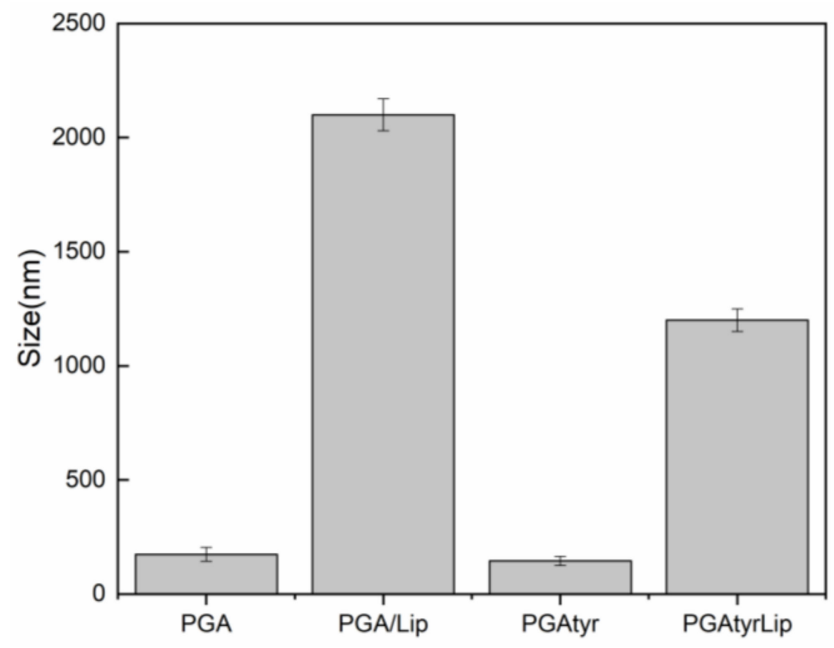

Figure 4. Size distribution and lipase assay (after $3 \mathrm{~h}$ of contact with the lipase). 


\subsection{Crystallinity, Contact Angle, and Microstructures of Films}

After the melting and crystallinity characterization of native starches, films were prepared by casting. Before any analysis, the films were equilibrated in an environment with controlled relative humidity (RH: 85\%). The crystallinity showed a decrease for all the films as compared to native starch (Table 1). In particular, the different crystalline polymorphs and their relative contribution to the overall crystallinity were altered following recrystallization during the drying process (Table 1). The crystallinity of the films was severely reduced by the heat treatment. In fact, the crystallinity was three-fold lower for the films with unmodified PGA NPs and four-fold lower when the NPs were grafted with tyrosine. Furthermore, for all films, no A-type polymorphism was individuated, confirming the complete melt of the crystalline structure during the heating treatment [5]. The crystalline polymorphs detected in the films are due to the natural process of recrystallization of the starch. Interestingly, the addition of NPCs in the starch films allowed the growth of a similar relative distribution of Vh- and B-type polymorphs.

Table 1. Percentage of crystallinity and the contact angle of native starch and cast films. Vh, B, and A denotes the crystalline polymorphs.

\begin{tabular}{cccccc}
\hline Sample & Cryst. (\%) & Vh (\%) & B (\%) & A (\%) & Contact Angle ( $\mathbf{~}^{\text {) }}$ \\
\hline StNative & 20 & 5 & 18 & 77 & \\
\hline StOn & 6 & 37 & 63 & 0 & $102 \pm 1$ \\
\hline StGl & 6 & 20 & 80 & 0 & $88 \pm 2$ \\
\hline StPGA3 & 7 & 52 & 48 & 0 & nd \\
\hline StPGA10 & 7 & 47 & 53 & 0 & $57 \pm 0.5$ \\
\hline StPGAtyr3 & 5 & 57 & 39 & 0 & nd \\
\hline StPGAtyr10 & 4 & 50 & 50 & 0 & $32 \pm 1$ \\
\hline
\end{tabular}

The stacked IR spectra of the four starch films (Figure 5) demonstrate that the introduction in the starch matrix of both glycerol and the polymeric NPs led to variations in the hydrogen bond network depicted by the variation in the wave number of the $\mathrm{OH}$ stretching in the region between 3600 and $3200 \mathrm{~cm}^{-1}$. In the spectra of starch mixed with pure PGA (StPGA) and starch mixed with the PGA modified with tyrosine (StPGATyr), the appearance of a peak in the region around $1700 \mathrm{~cm}^{-1}$ is noticeable. This band is related to the stretching of the ester group, confirming the blending of the NPs within the film. Minor peak shifting and splitting can be observed in the area around 3000 to $2800 \mathrm{~cm}^{-1}$ and 1050 to $950 \mathrm{~cm}^{-1}$ related to the $\mathrm{CH}$ stretching and the $\mathrm{C}-\mathrm{O}-\mathrm{C}$ stretching, respectively. Due to the tight hydrogen bond network among the hydroxyl groups in the starch molecules, an extremely smooth and hydrophobic surface was observed (Figure 6 and Table 1). This phenomenon is likely due to the masking of the polar $\mathrm{OH}$, involved in the intra- and intermolecular $\mathrm{H}$-bonding, within the starch matrix. However, by adding glycerol, PGA, or PGATyr NPs, a change in the recrystallization processes was expected and hence a perturbation of this $\mathrm{H}$-bonding network. The addition of these molecules resulted in a drop in the value of the water contact angle (Table 1). The variation in the water contact angle was more prominent after the addition of the two polymeric NPs compared to the variation related to the presence of glycerol (Table 1). Interestingly, a direct comparison of the water contact angle and smoothness was observed (Figure 6 and Table 1). The films with only starch (StOn) and starch mixed with glycerol (StGl) showed a smooth surface and consequently high contact angle values of $102^{\circ}$ and $88^{\circ}$, respectively. On the other hand, the addition of the polymeric NPs tremendously affected the integrity of the starch surface film (Figure 6). A remarkable drop in the water contact angle $\left(57^{\circ}\right.$ and $\left.32^{\circ}\right)$ can be ascribed to these superficial alterations. It can be speculated that both the aliphatic and aromatic hydroxyl groups in the starch films containing PGAtyr NPs (StPGATyr) may deeply alter 
the nature and arrangement of the H-bonding network within the film. The films containing StPGATyr typically showed a lower relative crystallinity.

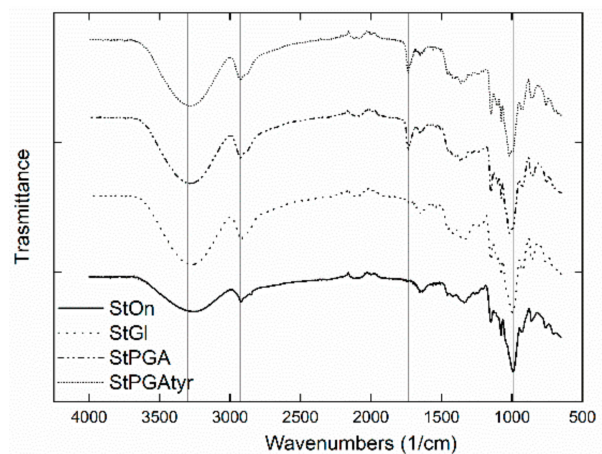

Figure 5. Stacked IR spectra of StOn, StGl, StPGA, and StPGATyr in the region $4000-650 \mathrm{~cm}^{-1}$.
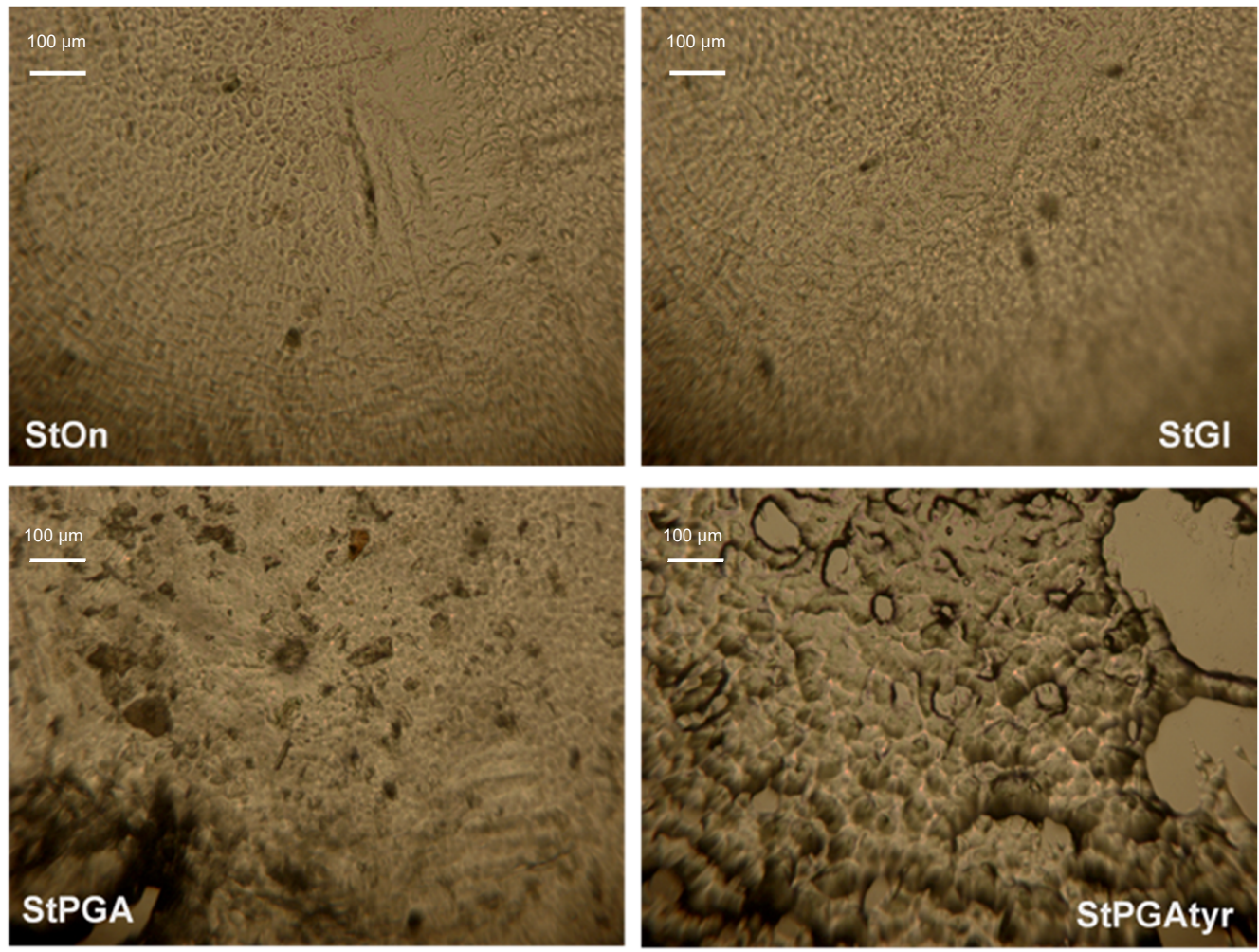

Figure 6. Optical microscopy pictures showing different surface smoothness of StOn, StGi, StPGA, and StPGATyr films.

To monitor the distribution of the poly(glycerol adipate) in the films, the NPs were loaded with a fluorescent dye (coumarin), which was dispersed into the films (Figure 7). Two phases were easily visualized. The films containing the 3\% NPs showed a fibril-like structure formed between starch (dark phase) and PGA (blue phase) (Figure 7B). This demonstrates that PGA NPs were stable during the drying of the films and the coumarin did not leak during fabrication and testing. Furthermore, the interaction between starch and PGA NPs was demonstrated. When the concentration of NPs was increased to $10 \%$, the fibril structure was lost, probably due to the aggregation of the NPs (Figure 7A). 

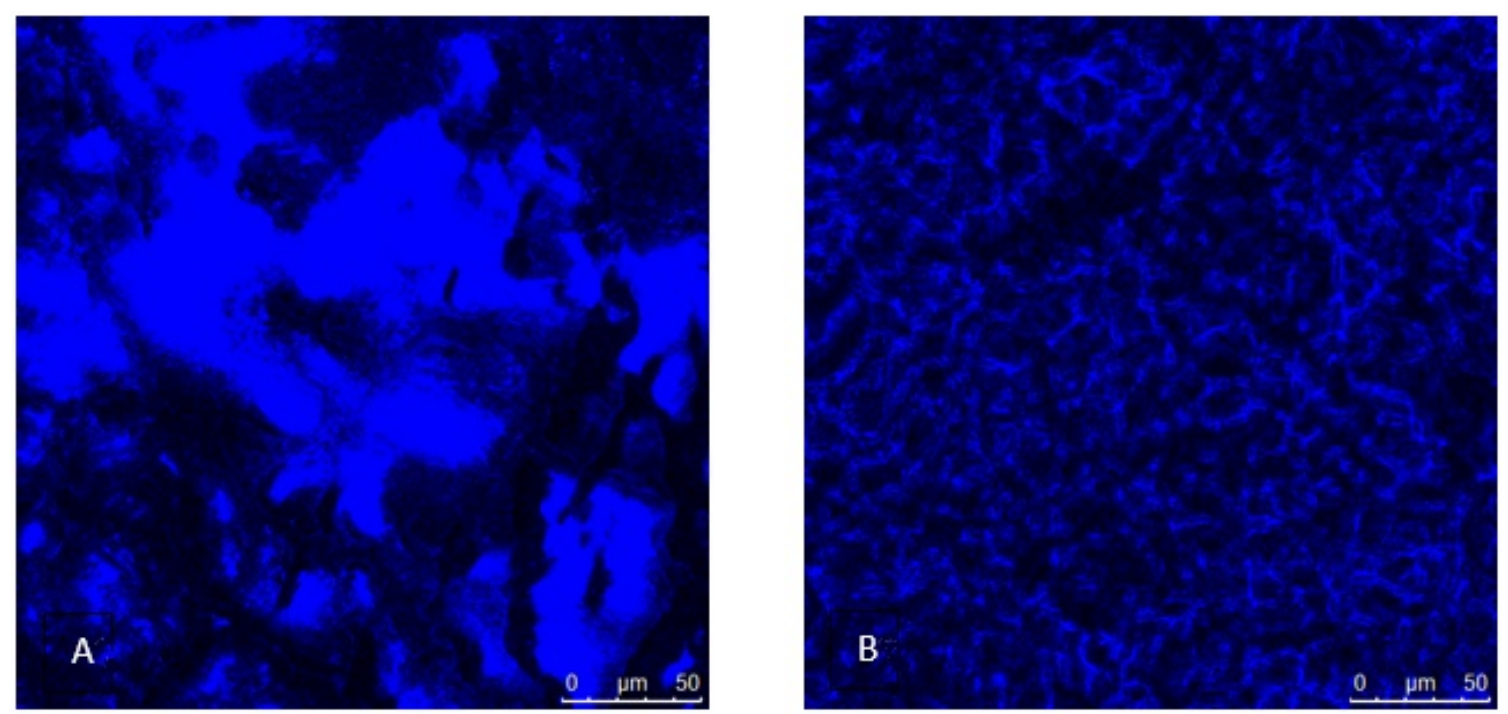

Figure 7. Confocal microscopy pictures showing the NPs distribution in the starch films. (A) StPGATyr 10\%; (B) StPGATyr 3\%.

\subsection{Thermo-Mechanical Properties}

Dynamic mechanical analysis was performed using controlled temperature and humidity scans. Firstly, the films were scanned to define the $\mathrm{Tg}$ of the blends equilibrated at $80 \% \mathrm{RH}$. In particular, the addition of NPCs showed a bimodal DMA trace distribution, with zones richer in NPs that vibrated at lower temperaturea. The starch-rich pockets showed a higher $\mathrm{Tg}$ as compared to the starch alone (Table 2). The glass transition temperature of free PGA could not be recorded in these experiments. This was caused by the fact that PGA assembled in NPs embedded in a complex three-dimensional and semi-crystalline matrix. Hence, the glass transition of the poly-ester changed under these conditions, increasing to about $37^{\circ} \mathrm{C}$.

Table 2. Glass transition temperature measured using a dynamic mechanical temperature analyser and dynamic mechanical humidity analyser.

\begin{tabular}{ccc}
\hline Film & Tg & Tg-RH \\
\hline starch & 66 & $37^{\circ} \mathrm{C}-80 \%$ \\
\hline StPGA3 & $38 /-$ & \\
\hline StPGA3tyr & $-/ 80$ & $37^{\circ} \mathrm{C}-57 \%$ \\
\hline StPGA10 & $37 / 80$ & \\
\hline StPGA10tyr & $37 / 80$ & \\
\hline
\end{tabular}

StGl and StPGATyr were further analyzed in a humidity-controlled environment (Figure 8). In particular, these results showed that the PGA NPs changed their sensitivity to the water of the starch films (Table 2). The glass transition of starch at $37^{\circ} \mathrm{C}$ was detected only when the relative humidity reached $80 \%$. On the other hand, StPGAtyr3 films showed a $\mathrm{Tg}$ transition at a relative humidity of $57 \%$ relative humidity, indicating a tendency in lowering the $\mathrm{Tg}$ in a lower-moisture environment. 

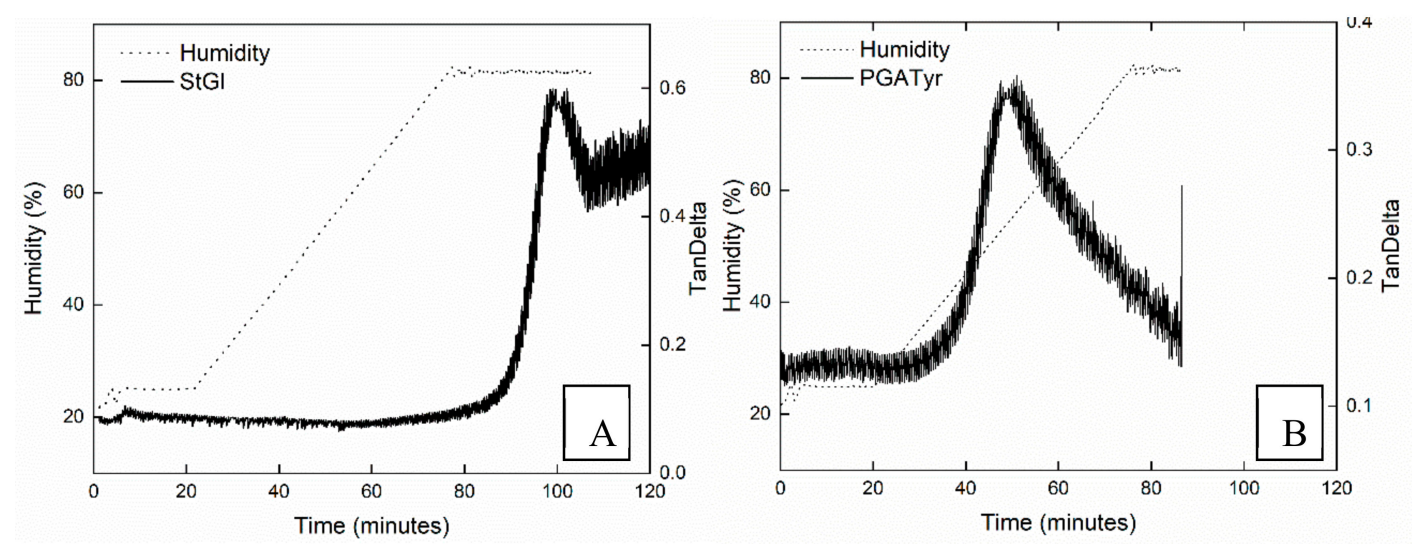

Figure 8. Dynamic mechanical analysis with humidity control of (A) starch film with $25 \%$ glycerol and (B) starch film with 25\% glycerol and 3\% PGA NPs.

\subsection{Biocompatibility Tests}

Because starch is a biopolymer digested in the human intestine, biocompatibility tests were performed on Caco-2 cells, thus giving insight into the possible utilization of these films as a biocompatible material for drug or bio-actives release.

Cytotoxicity analysis in Caco-2 cells of film extracts generated from different incubation times with culture medium demonstrated that the extracts from the materials can be considered biocompatible, as no loss in cellular metabolic activity or damage to cell membranes was experienced following incubation with cells for $48 \mathrm{~h}$ (Figure 9). Indeed, compared to the negative control (DMEM), increases in metabolic activity (approximately $10 \%$ to $20 \%$ ) were observed in various groups, including the starch and StGl and StOn films (Figure 9); an effect likely related to the presence of free starch in the film extracts and the subsequent availability of this carbohydrate for cellular metabolism.
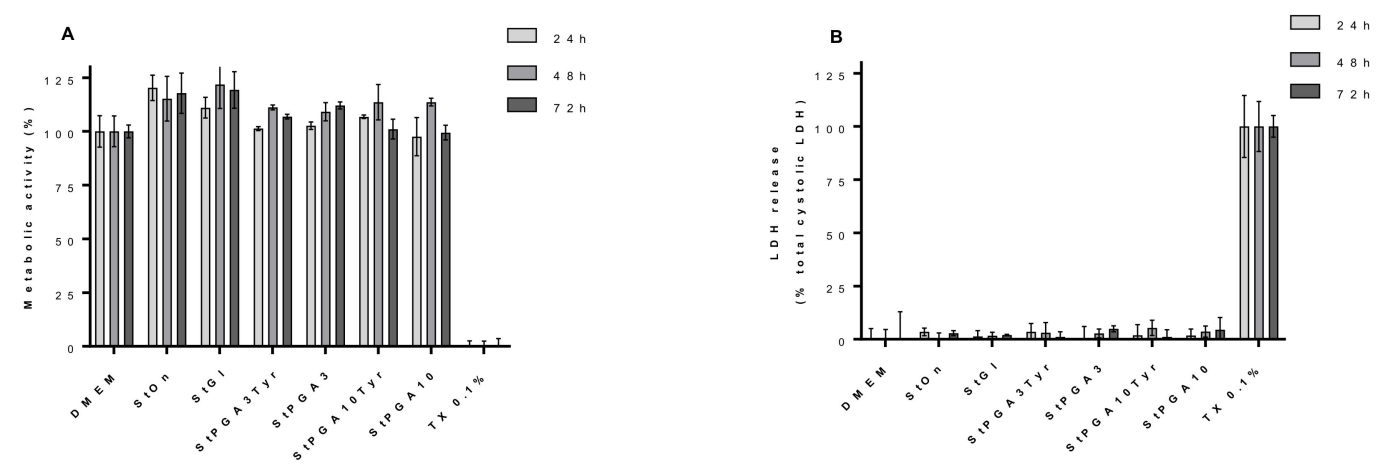

Figure 9. Cytocompatibility of PGA films assessed via (A) cellular metabolic activity and (B) LDH release in Caco-2 intestinal epithelial cells. Films were incubated with DMEM ( $3 \mathrm{~cm}^{2}$ per $\left.\mathrm{mL}\right)$ for 24 , 48 , or $72 \mathrm{~h}$ and the resulting solutions were applied to cells for $48 \mathrm{~h}$. Data are presented as mean \pm S.D $(n=3)$.

These data demonstrate that starch-based films can be used as a base for biomaterials and for drug delivery. In fact, the films containing NPs did increase the metabolic activity.

\section{Conclusions}

Casted barley starch films were tested as bio polymeric carriers for poly-(glycerol adipate)-based nanoparticles. The native starch granules showed typical behavior during characterization of the melting profile and crystallinity. When the films were prepared, the overall crystallinity varied. In fact, for all the films, we identified a decrease in crystallinity down to a minimum of $4 \%$. Furthermore, the films showed a mixture of Vh- and B-type polymorphs. Interestingly, the films with 
NPs showed a ratio between B and Vh of approximately 1:1. This result may be an indication of the formation of starch/PGA complexes.

The integration of nanoparticles in glycerol-plasticized films showed an interesting effect on the glass transition temperature. In fact, it was possible to measure variation in $\mathrm{Tg}$ and an increase in moisture sensitivity as well as in the final wettability of the film surface and superficial roughness.

In conclusion, the films showed a good capability for stimulating the growth of Caco-2 cells. Our work proves that starch has the potential to be a good biocompatible material and a coating polymer for nanoparticles. Furthermore, the films created are a possible alternative as a coating for food materials and the delivery of bioactive molecules (e.g., flavors).

Author Contributions: Conceptualization, D.S., V.T.; data curation, D.S., R.C., J.D., and V.T.; formal analysis, D.S., R.C., J.D., and V.T.; funding acquisition, D.S.; investigation, D.S., R.C., J.X., S.M.E.S., J.D., and V.T.; supervision, S.M.H.; writing—original draft, D.S. and V.T.; writing—review and editing, D.S., A.B., V.T., and A.M.H.

Funding: This research was funded by Danish council for independent research, grant number 7026-00060B.

Acknowledgments: The authors thank the Danish Council for Independent Research Technology, for the invaluable help guiding me towards this work. The authors also thank the Center for Advanced Bioimaging, Faculty of Science, the University of Copenhagen for use of their microscopy facilities.

Conflicts of Interest: The authors declare no conflict of interest. The funders had no role in the design of the study; in the collection, analyses, or interpretation of data; in the writing of the manuscript, or in the decision to publish the results.

\section{References}

1. Pérez, S.; Bertoft, E. The molecular structures of starch components and their contribution to the architecture of starch granules: A comprehensive review. Starch/Staerke 2010, 62, 389-420. [CrossRef]

2. Blennow, A.; Engelsen, S.B.; Nielsen, T.H.; Baunsgaard, L.; Mikkelsen, R. Starch phosphorylation: A new front line in starch research. Trends Plant Sci. 2002, 7, 445-450. [CrossRef]

3. Blennow, A.; Mette Bay-Smidt, A.; Bauer, R. Amylopectin aggregation as a function of starch phosphate content studied by size exclusion chromatography and on-line refractive index and light scattering. Int. J. Biol. Macromol. 2001, 28, 409-420. [CrossRef]

4. Sagnelli, D.; Kirkensgaard, J.J.K.; Giosafatto, C.V.L.; Ogrodowicz, N.; Kruczał, K.; Mikkelsen, M.S.; Maigret, J.E.; Lourdin, D.; Mortensen, K.; Blennow, A. All-natural bio-plastics using starch-betaglucan composites. Carbohydr. Polym. 2017, 172, 237-245. [CrossRef] [PubMed]

5. Sagnelli, D.; Hebelstrup, K.H.; Leroy, E.; Rolland-Sabaté, A.; Guilois, S.; Kirkensgaard, J.J.K.; Mortensen, K.; Lourdin, D.; Blennow, A. Plant-crafted starches for bioplastics production. Carbohydr. Polym. 2016, 152, 398-408. [CrossRef] [PubMed]

6. Sagnelli, D.; Hooshmand, K.; Kemmer, G.C.; Kirkensgaard, J.J.K.; Mortensen, K.; Giosafatto, C.V.L.; Holse, M.; Hebelstrup, K.H.; Bao, J.; Stelte, W.; et al. Cross-Linked Amylose Bio-Plastic: A Transgenic-Based Compostable. Int. J. Mol. Sci. 2017, 18, 2075.

7. Lourdin, D.; Coignard, L.; Bizot, H.; Colonna, P. Influence of equilibrium relative humidity and plasticizer concentration on the water content and glass transition of starch materials. Polymer (Guildf) 1997, 38, 5401-5406. [CrossRef]

8. Follain, N.; Joly, C.; Dole, P.; Bliard, C. Mechanical properties of starch-based materials. I. Short review and complementary experimental analysis. J. Appl. Polym. Sci. 2005, 97, 1783-1794. [CrossRef]

9. Montero, B.; Rico, M.; Rodríguez-Llamazares, S.; Barral, L.; Bouza, R. Effect of nanocellulose as a filler on biodegradable thermoplastic starch films from tuber, cereal legume. Carbohydr. Polym. 2017, 157, 1094-1104. [CrossRef] [PubMed]

10. Jantanasakulwong, K.; Leksawasdi, N.; Seesuriyachan, P.; Wongsuriyasak, S.; Techapun, C.; Ougizawa, T. Reactive blending of thermoplastic starch, epoxidized natural rubber and chitosan. Eur. Polym. J. 2016, 84, 292-299. [CrossRef]

11. Kallinteri, P.; Higgins, S.; Hutcheon, G.A.; St. Pourçain, C.B.; Garnett, M.C. Novel functionalized biodegradable polymers for nanoparticle drug delivery systems. Biomacromolecules 2005, 6, 1885-1894. [CrossRef] [PubMed] 
12. Taresco, V.; Creasey, R.G.; Kennon, J.; Mantovani, G.; Alexander, C.; Burley, J.C.; Garnett, M.C. Variation in structure and properties of poly(glycerol adipate) via control of chain branching during enzymatic synthesis. Polymer (Guildf) 2016, 89, 41-49. [CrossRef]

13. Taresco, V.; Suksiriworapong, J.; Styliari, I.D.; Argent, R.H.; Swainson, S.E.; Booth, J.; Turpin, E.; Laughton, C.A.; Burley, J.C.; Alexander, C.; et al. New N-acyl amino acid-functionalized biodegradable polyesters for pharmaceutical and biomedical applications. RSC Adv. 2016, 6, 109401-109405. [CrossRef]

14. Bilal, M.H.; Hussain, H.; Prehm, M.; Baumeister, U.; Meister, A.; Hause, G.; Busse, K.; Mäder, K.; Kressler, J. Synthesis of poly(glycerol adipate)-g-oleate and its ternary phase diagram with glycerol monooleate and water. Eur. Polym. J. 2017, 91, 162-175. [CrossRef]

15. Wersig, T.; Krombholz, R.; Janich, C.; Meister, A.; Kressler, J.; Mäder, K. Indomethacin functionalised poly(glycerol adipate) nanospheres as promising candidates for modified drug release. Eur. J. Pharm. Sci. 2018, 123, 350-361. [CrossRef] [PubMed]

16. Taresco, V.; Suksiriworapong, J.; Creasey, R.; Burley, J.C.; Mantovani, G.; Alexander, C.; Treacher, K.; Booth, J.; Garnett, M.C. Properties of acyl modified poly(glycerol-adipate) comb-like polymers and their self-assembly into nanoparticles. J. Polym. Sci. Part A Polym. Chem. 2016, 54, 3267-3278. [CrossRef] [PubMed]

17. Suksiriworapong, J.; Taresco, V.; Ivanov, D.P.; Styliari, I.D.; Sakchaisri, K.; Junyaprasert, V.B.; Garnett, M.C. Synthesis and properties of a biodegradable polymer-drug conjugate: Methotrexate-poly(glycerol adipate). Colloids Surf. B Biointerfaces 2018, 167, 115-125. [CrossRef]

18. Wang, M.O.; Etheridge, J.M.; Thompson, J.A.; Vorwald, C.E.; Dean, D.; Fisher, J.P. Evaluation of the in vitro cytotoxicity of cross-linked biomaterials. Biomacromolecules 2013, 14, 1321-1329. [CrossRef]

19. Vasanthan, T.; Hoover, R. Barley Starch: Production, Properties, Modification and Uses. In Starch; Elsevier: Amsterdam, The Netherlands, 2009; pp. 601-628.

20. Marin, E.; Briceño, M.I.; Caballero-George, C. Critical evaluation of biodegradable polymers used in nanodrugs. Int. J. Nanomed. 2013, 8, 3071-3091. [PubMed]

21. Colombo, C.; Dragoni, L.; Gatti, S.; Pesce, R.M.; Rooney, T.R.; Mavroudakis, E.; Ferrari, R.; Moscatelli, D. Tunable degradation behavior of PEGylated polyester-based nanoparticles obtained through emulsion free radical polymerization. Ind. Eng. Chem. Res. 2014, 53, 9128-9135. [CrossRef] 